\title{
A note on Hadamard arrays
}

\section{Joan Cooper}

Let $v=m k+1$ be a prime power; we show for $m$ even it is not possible to partition the Galois field $G F(v)$ to give four $(0,1,-1)$ matrices $x_{1}, x_{2}, x_{3}, x_{4}$ satisfying:

(i) $x_{i} * x_{j}=0, i \neq j, i, j=1,2,3,4$;

(ii) $\sum_{i=1}^{4} x_{i}$ is a $(1,-1)$ matrix;

(iii) $\sum_{i=1}^{4} X_{i} X_{i}^{T}=v I_{v}$.

Thus this method of partitioning the Galois field GF(v), into four matrices satisfying the above conditions, cannot be used to find Baumert-Hall Hadamard arrays $\mathrm{BH}[4 v]$ for $v=9,11,17,23,27,29, \ldots$.

\section{Terminology and definitions}

A $4 n \times 4 n$ Hadamard array, $H$, is a square matrix of order $4 n$ with elements $\pm A, \pm B, \pm C, \pm D$ each repeated $n$ times in each row and column. Assuming the indeterminants $A, B, C, D$ comute, the row vectors of $H$ must be orthogonal.

The Hadamard product, $\star$, of two matrices $A=\left(a_{i j}\right)$ and $B=\left(b_{i j}\right)$ which are the same size is given by

$$
A \star B=\left(a_{i, j} b_{i j}\right)
$$

The identity matrix will be represented as $I$ and the $v \times v$ matrix Received 1 August 1973. Communicated by Jennifer R.S. Wallis. 
of all l's will be $J$.

The symbol \& represents the result from adjoining two sets with repetition remaining; that is,

$$
\left\{x_{1}, \ldots, x_{s}\right\} \&\left\{y_{1}, \ldots, y_{t}\right\}=\left[x_{1}, \ldots, x_{s}, y_{1}, \ldots, y_{t}\right] .
$$

Where repetition occurs the elements resulting from such an adjunction will be called a collection and denoted by square brackets [ ].

A binary composition $\wedge$ of two sets will be defined as

$$
\begin{aligned}
A_{1} \wedge A_{2} & =\left[x_{1}, \ldots, x_{s}\right] \wedge\left[y_{1}, \ldots, y_{t}\right] \\
& =\left[x_{1}+A_{2}, \ldots, x_{s}+A_{2}\right] .
\end{aligned}
$$

Let $v=m k+1=p^{\alpha}$ (a prime power). Let $x$ be a primitive element of $F=G F(v)$ and write $G=\left\{z_{1}, \ldots, z_{v-1}\right\}$ for the multiplicative cyclic group of order $v-1$ generated by $x$.

Choose the cosets $C_{i}$ of $G$ by

$$
c_{i}=\left\{x^{k j+i}: 0 \leq j \leq m-1\right\} \quad 0 \leq i \leq k-1 \text {, }
$$

where the order of $c_{i}$ is $m$ and its index $k$.

Now let $D_{i}=\left(d_{j l}\right)$ be the incidence matrix of the $\operatorname{coset} c_{i}$. $D_{i}=\left(d_{j l}\right)$ is defined as

$$
d_{j l}= \begin{cases}1 & \text { if } z_{l^{-z}} \in C_{i}, \\ 0 & \text { otherwise. }\end{cases}
$$

We will denote $D_{i}$ by $\left[C_{i}\right]$.

As $G=C_{0} \cup C_{1} \cup \ldots \cup C_{k-1}=F \backslash\{0\}$, its incidence matrix is $J-I$ and the incidence matrix of $F$ is $J$.

Therefore the incidence matrix of $\{0\}$ will be $I$.

$X=\left[\begin{array}{ccc}k-1 & & \\ \& & b_{s} C_{s}\end{array}\right]$ will mean the matrix $X$ which is a summation of the incidence matrices of the cosets. That is 
(1)

$$
x=\left[\begin{array}{ccc}
k-1 & \\
\sum_{8=0} & b_{8} C_{8}
\end{array}\right]=\sum_{s=0}^{k-1} b_{8}\left[c_{s}\right],
$$

$b_{\boldsymbol{g}} \in Z$, the integers. Note from the definition of a binary composition

$$
\text { \{0\}^ } c_{i}=c_{i} \text {. }
$$

We will define the transpose of a coset $C_{i}^{T}$ by:

$$
\begin{aligned}
& c_{i}=\left\{x^{k j+i}: 0 \leq j \leq m-1\right\}, \\
& c_{i}^{T}=\left\{-x^{k j+i}: 0 \leq j \leq m-1\right\} .
\end{aligned}
$$

LEMMA 1 [1]. If $m$ is even, $C_{i}^{T}=C_{i}$; and if $m$ is odd, $c_{i}^{T}=c_{i+\frac{1}{k} k}$.

THEOREM 2 [1]. If $c_{i}$ and $c_{i}$ are two cosets of order $m$ and index $k$ of the group $G$, then the binary composition of $c_{i}$ and $c_{2}$ is given by:

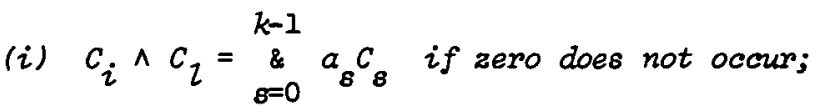

$$
\begin{aligned}
& \text { (ii) } c_{i} \wedge c_{2}=m\{0\} \& \underset{s=0}{k-1} a_{s} C_{s} \text { if zero does occur; }
\end{aligned}
$$

where the $a_{8}$ are integers giving multiplicities.

LEMMA 3. If

(i) zero does not occur in $C_{i} \wedge C_{l}$ then

$$
\sum_{B=0}^{k-1} a_{B}=m ;
$$

(ii) zero does occur in $c_{i} \wedge C_{i}$ then

$$
\sum_{s=0}^{k-1} a_{8}=m-1
$$


LEMMA 4 [1]. $\quad c_{i} \wedge C_{2}=m[0\} \& \&_{s=0}^{k-1} a_{s} C_{s}$ if and on $z_{y}$ if $c_{2}=c_{i}^{T}$.

LEMMA 5 [1]. If

(i) $c_{i} \neq C_{i}^{T}$ in $c_{i} \wedge c_{i}$ then

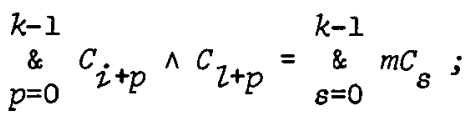

(ii) $C_{2}=C_{i}^{T}$ in $C_{i} \wedge C_{i}$ then

$$
\sum_{p=0}^{k-1} C_{i+p} \wedge C_{Z+p}=k m\{0\} \& \sum_{s=0}^{k-1}(m-1) C_{s} .
$$

\section{Method of partitioning $G F(v)$}

The incidence matrices $\left[C_{i}\right]$ of the cosets $C_{i}$ and the identity matrix $I$ are partitioned into four $(0,1,-1)$ matrices $x_{1}, x_{2}, x_{3}, x_{4}$ such that

$$
\begin{gathered}
x_{i} * X_{j}=0, \quad i \neq j, i, j=1,2,3,4 ; \\
\sum_{i=1}^{4} X_{i} X_{i}^{T}=v I_{v} .
\end{gathered}
$$

We show for $m$ even with $X_{i} * X_{j}=0$ it is not possible to get $\sum_{i=1}^{4} x_{i} x_{i}^{T}=v I_{v}$

THEOREM 6. Let $v=m k+1=p^{\alpha}$ ( $p$ a prime) with $m$ even. Further suppose $c_{i}$ are cosets of order $m$ defined above.

Let

$$
x_{i}=\left[\begin{array}{cc}
k-1 & \\
\&_{s=0}^{\&} & a_{i s} c_{s}
\end{array}\right], \quad i=1,2,3,4,
$$

and suppose exactly one of $x_{1_{s}}, a_{2}, a_{3_{s}}, a_{4_{s}}$ is 1 or -1 and $I$ 
Hadamard arrays

19

belongs to one of the $x_{i}$ 's.

Then

$$
\sum_{i=1}^{4} X_{i} X_{i}^{m}=v I_{v}
$$

is not possible.

Proof. Without loss of generality let $I$ occur in $X_{1}$.

$$
\begin{aligned}
& X_{1}=\left[\begin{array}{ccccc}
k-1 & & \\
\&_{s=0} & a_{1} & c_{s} & \{0\}
\end{array}\right] \\
& =\sum_{\varepsilon=0}^{k-1} a_{1_{s}}\left[C_{s}\right]+I \text { from (1); }
\end{aligned}
$$

for $i=2,3,4$,

$$
x_{i}=\sum_{s=0}^{k-1} a_{i_{s}}\left[C_{s}\right] .
$$

Since $m$ is even from Lemma 1 ,

$$
C_{i}^{T}=C_{i}
$$

thus $X_{i} X_{i}^{T}$ becomes $x_{i}^{2}$ for all $i$ and we have

$$
\begin{aligned}
& x_{1}^{2}=\left(\sum_{s=0}^{k-1} a_{1_{s}}\left[C_{s}\right]+I\right)^{2}, \\
& x_{i}^{2}=\left(\sum_{s=0}^{k-1} a_{i_{s}}\left[c_{s}\right]\right)^{2}, i \neq 1, \\
& x_{1}^{2}=\sum_{s=0}^{k-1} a_{1_{s}}^{2}\left[C_{s}\right]^{2}+2 \sum_{s=0}^{k-1} \sum_{p=s+1}^{k-1} a_{1_{s}} a_{I_{p}}\left[C_{s}\right]\left[C_{p}\right]+2 \sum_{s=0}^{k-1} a_{1_{s}}\left[C_{s}\right]+I .
\end{aligned}
$$

For $i=2,3,4$,

$$
x_{i}^{2}=\sum_{s=0}^{k-1} a_{i_{s}}^{2}\left[C_{s}\right]^{2}+2 \sum_{s=0}^{k-1} \sum_{p=s+1}^{k-1} a_{i_{s}} a_{i_{p}}\left[C_{s}\right]\left[C_{p}\right] .
$$

Now

https://doi.org/10.1017/S0004972700040570 Published online by Cambridge University Press 


$$
\begin{aligned}
\sum_{i=1}^{4} x_{i} x_{i}^{T} & =\sum_{i=1}^{4} x_{i}^{2} \\
= & \sum_{s=0}^{k-1}\left[c_{s}\right]^{2} \text { from the conditions of the theorem } \\
& +2 \sum_{i=1}^{4}\left(\sum_{s=0}^{k-1} \sum_{p=s+1}^{k-1} a_{i s} a_{i p}\left[C_{s}\right]\left[c_{p}\right]\right)+2 \sum_{s=0}^{k-1} a_{1_{s}}\left[C_{s}\right]+I ;
\end{aligned}
$$

$\sum_{i=1}^{4} x_{i}^{2}=k m I+(m-1) \sum_{s=0}^{k-1}\left[c_{s}\right]$ from Lemma 5

$$
+2 \sum_{i=1}^{4}\left(\sum_{\varepsilon=0}^{k-1} \sum_{p=s+1}^{k-1} a_{i s} a_{i p}\left(\sum_{j=0}^{k-1} b_{j}\left[c_{j}\right]\right)\right) \text { by Theorem } 2(i)
$$$$
+2 \sum_{8=0}^{k} a_{1_{s}}\left[c_{s}\right]+I
$$

(2)

$$
\sum_{i=1}^{4} x_{i}^{2}=(k m+1) I+(m-1) \sum_{s=0}^{k-1}\left[c_{s}\right]+2 \sum_{j=0}^{k-1} d_{j}\left[c_{j}\right] \text {, }
$$

where $d_{j}$ comes from collecting all the cosets together from the third and fourth terms of the equation above.

It can be easizy seen that it is not possible to get $\sum_{i=1}^{4} x_{i}^{2}=v I v$ as $m-1$ is odd and the 2 in front of the last term of equation (2) gives all the cosets from this term an even number of times.

For $v=9,11,17, \ldots, m$ cannot be odd, by a result in [2]. We have just shown $m$ cannot be even. So it is impossible to partition $\mathrm{GF}(v)$ by the method of [2] in order to construct Hadamard arrays, for those values of $v$.

\section{References}

[1] Joan Cooper, "A binary composition for collections and sets", Proc. First Austral. Conf. Combinatorial Math., Newcastle, 1972, 145-161 (TUNRA, Newcastle, 1972). 
[2] Joan Cooper and Jennifer Wallis, "A construction for Hadamard arrays", Bull. Austral. Math. Soc. 7 (1972), 269-277.

[3] David C. Hunt and Jennifer Wallis, "Cyclotomy, Hadamard arrays and supplementary difference sets", Proc. Second Manitoba Conf. Numerical Mathematics, October 1972, 351-381 (Congressus Numerantium, 7. University of Manitoba, Winnipeg, 1972).

[4] Thomas Storer, Cyclotomy and difference sets (Lectures in Advanced Mathematics, 2. Markham, Chicago, Illinois, 1967).

Department of Mathematics, University of Newcastle, Newcastle,

New South Wales. 